\title{
Magnet End Design: The Main Injector Dipoles
}

\author{
J. Ostiguy \\ Fermi National Accelerator Laboratory \\ P.O. Box 500, Batavia, Illinois 60510
}

August 1991

* Presented at the 14th Biennial IEEE Particle Accelerator Conference, San Francisco, CA, May 6-9, 1991. 


\section{Disclaimer}

This report was prepared as an account of work sponsored by an agency of the United States Government. Neither the United States Government nor any agency thereof, nor any of their employees, makes any warranty, express or implied, or assumes any legal liability or responsibility for the accuracy, completeness, or usefullness of any information, apparatus, product, or process disclosed, or represents that its use would not infringe privately owned rights. Reference herein to any specific commercial product, process, or service by trade name, trademark, manufacturer, or otherwise, does not necessarily constitute or imply its endorsement, recommendation, or favoring by the United States Government or any agency thereof. The views and opinions of authors expressed herein do not necessarily state or reflect those of the United States Government or any agency thereof. 


\title{
Magnet End Design: The Main Injector Dipoles
}

\author{
Jean-François OSTIGUY \\ Fermilab, P.O. Box 500, Batavia, IL 60510
}

\section{Abstract}

From the accelerator designer standpoint, one of the quantities of interest in a magnet is the axially integrated transverse field. It is easily shown that the latter satisfies the equations of $2 \mathrm{D}$ magnetostatics. This is the basic theoretical result needed to design accelerator magnet ends. Unfortunately, axially integrated fields must be obtained from accurate 3D field maps and magnets ends have historically been designed using a cut and try approach. To a certain extent, this remains true even today; however, the advent of reliable 3D magnet design codes now permits to substantially reduce the costs associated with the construction of various prototypes. In this paper, the theory of magnet end design is reviewed. The design of the end of the dipole magnets of the proposed Fermilab Main Injector is analyzed in a detailed manner using TOSCA, a well-established 3D finite element code. Provided the limitations of the code are well understood by the user, the integrated field profile is satisfactorily predicted.

\section{Theory}

The relevant data for particle tracking is the net amount $\Delta p$ of momentum transferred from the longitudinal to the tranverse degrees of freedom of a particle during its passage through a magnet. Assuming the wavelength of the betatron oscillations is sufficiently large compared with the length of the magnet, one can consider the trajectories of the charged particles to be straight lines. For such trajectories,

$$
\begin{aligned}
\Delta \mathbf{p} & =\int_{-\infty}^{+\infty} q \hat{\mathbf{v}} \times \mathbf{B}(x, y, s) \frac{1}{v} d s \\
& \simeq q\left[-\bar{B}_{y} \hat{\mathbf{x}}+\bar{B}_{x} \hat{\mathbf{y}}\right]
\end{aligned}
$$

where the overbar denotes $z$-integrated quantities. Here $s$ is the longitudinal position along the closed orbit; $x$ and $y$ are the transverse coordinates, $x$ being in the deflecting plane. To a very good approximation, one has $s \simeq z$. As usual, $q$ is the charge and $\mathbf{v}$ is the velocity of the particle. In the source-free aperture of a magnet, the magnetostatic field can be represented by a harmonic scalar potential $\Phi(x, y, z)$. Integrating Laplace's equation in cartesian coordinates with respect to $z$ leads to the relation

$$
\nabla_{t} \bar{\Phi}(x, y)=-\left.\frac{\partial \Phi}{\partial z}\right|_{z_{2}}--\left.\frac{\partial \Phi}{\partial z}\right|_{z_{1}}=0
$$

where $\nabla_{t}$ is the transverse Laplacian. This is a remarkable result: the $z$-integrated potential $\dot{\Phi}(x, y)$ is a harmonic function if $z_{1}$ and $z_{2}$ are chosen in such a way that

$$
B_{z}\left(x, y, z_{2}\right)=B_{z}\left(x, y, z_{1}\right)
$$

* Operated by the Universitjes Research Association, Inc., under contract with the U.S. Department of Energy.
This constraint is certainly satisfied $z_{1}$ and $z_{2}$ are at infinity. If the magnet geometry is such that $B_{z}$ has odd symmetry with respect to $z$, one could also choose one of the limits to coincide with the origin. In practice, the condition (4) can only be satisfied approximately; the point $z_{1}$ is usually a point well inside the body of the magnet while the point $z_{2}$ is away from the edge of the magnet in a region where $\mathbf{B}$ (and a fortiori $B_{z}$ ) is expected to be very small.

The fact that the integrated potential is harmonic implies that the integrated transverse magnetic field has all the properties of a two-dimensional field. In particular, for a magnet that has mirror symmetry with respect to the horizontal and vertical midplane, the integrated field over the entire aperture is completely determined by the values of the vertical field on the $x$-axis. An end correction scheme can therefore be designed on the sole basis of its effect on the integrated field in the horizontal midplane.

\section{D Magnetostatics Codes}

The numerical solution of the equations of magnetostatics in 3D can be approached in a different number of ways. GFUN, one of the first widely available 3D codes, attacked the problem by constructing an integral equation for the magnetization. Essentially, the field produced by the coils is first computed by numerical integration using Ampere's formula. The induced magnetization is then calculated and iterations are performed until the relation between $\mathbf{M}$ and $\mathbf{H}$ becomes self-consistent.

This method has two advantages: (1) only the regions which contain magnetic materials need to be discretized (2) no spurious boundary conditions need to be imposed to simulate open boundaries. Unfortunately, the GFUN algorithm has a serious drawback: at each iteration, the field due to the induced magnetization is computed by summing the field of a distribution of magnetic dipole in free space. Since the field must also be computed separately at every single point of the grid, the computational cost entailed is prohibitive.

In current state-of-the-art 3D magnet design codes, the magnetic field is split into a solenoidal component $\mathbf{B}_{s}$ and an irrotational component $\mathbf{B}_{\boldsymbol{i}}$. The solenoidal component is still obtained by applying Ampere's formula to the conductors. This choice corresponds to the imposition of particular boundary conditions on $\mathbf{B}_{s}$. These conditions, combined with the boundary conditions prescribed for $\mathbf{B}$, determine the boundary conditions on $\mathbf{B}_{i}$. Since $\mathbf{B}_{s}$ is fixed, $\mathbf{B}_{i}$ can be represented by a reduced scalar potential $\phi . \phi$ is then computed using the finite element method.

In practice, $\mathbf{B}_{\boldsymbol{s}}$ and $\mathbf{B}_{\boldsymbol{i}}$ often tend to be of approximately equal magnitudes and opposite directions in highly permeable materials; this may lead to serious errors on $\mathbf{B}$. The difficulty can be side-stepped by using a total scalar potential in regions where cancellation errors are expected to occur. This amounts to choose $\mathbf{B}_{s}=0$ in these re- 
gions where, of course, no current can be allowed to circulate. The code TOSCA [1] which we are currently using at Fermilab was the first one to implement the two-scalar potential formulation [1]. TOSCA started as a research project at the Rutherford Laboratory, U.K.; it is now a well-established and supported commercial product.

It is worth noting in passing that it is possible to represent the magnetic field by a vector potential $\mathbf{A}$ to obtain a numerical solution. This formulation offers the advantage of eliminating the need to compute the coil fields by integration. Unfortunately, the imposition of a gauge on A poses some technical problems. Recent advances will probably change this state of affair and commercial codes based on a vector potential formulation should become more commonplace in a near future.

\section{End Pack Design}

Because of their length, dipole magnets are often considered to be quasi two-dimensional objects. Indeed, the body fields can be predicted to a part in $10^{4}$ using a conventional two-dimensional code. To the extent that end effects can be considered as a small perturbation, the first generation of accelerator magnets was build without paying special attention to them. The body of the magnet terminated abruptly and that was it. The principal problem with such "sharp ends" is that the flux concentrates in the vicinity of the iron edge. As a result, the edge region saturates prematurely and the integrated dipole field tends to depend on the excitation current. The well-known cure for this problem is a beveled end.

Due to fringing, the vertical field in the midplane is stronger on the axis and gets weaker as one moves horizontally, away from the axis. For this reason, the integrated field of an uncorrected end exhibits rather strong negative (with respect to the dipole component) sextupole and decapole components. The correction scheme must enhance the integrated field for paths which are away from the axis in the horizontal plane. This is achieved in the current design by the addition of four shims, as shown in figure 1. Note that the exact shape of the shims is not critical; the basic rule is to avoid acute angles. In that respect, smooth curved surfaces would be ideal; however, the end packs have to be easy to fabricate.

The main injector dipole magnets come in two varieties. The so-called full length dipoles are designed to have an effective length of 6.096 meters ( 240 in). The second type of dipole magnet is identical to the first one, except that it has $2 / 3$ of the effective length. The magnets are curved with a sagitta of $1.598 \mathrm{~cm}(0.629 \mathrm{in})$ and have parallel faces. The body of the magnet is 5.8013 meters long (228.4 in) and is terminated by two removable $14.9225 \mathrm{~cm}$ (5.875 in) long end packs.

Because of saturation, the field quality (i.e. its deviation from uniformity) of a dipole magnet depends on the excitation current. Due to the length of the magnet, the variations in the quality of the axially integrated field is mostly determined by the $2 \mathrm{D}$ body field. The contribution of the end packs, which together account for roughly $5 \%$ of the effective length of a full-length dipole, remains relatively constant. Clearly, it is not possible for the end to compensate for the imperfections in the body field at all currents. The design objective was therefore to design "neutral" end packs. In other words, the contribution of the ends to the integrated field quality had to be as small as possible.

\section{Results}

Using the code TOSCA, three types of ends were modeled: a sharp end, a beveled end and a beveled end with shims. Due to a practical limitation of about 50,000 nodes, the total length of the simulated magnets was set to $20.32 \mathrm{~cm}$. This corresponds to the actual physical length of both end packs. The field was represented by a total scalar potential in the gap region and by a reduced potential in the region in front of the bevel. Spurious Neumann boundary conditions were imposed on the reduced potential at $z=50 \mathrm{~cm}$ and at $x=y=30 \mathrm{~cm}$ to simulate open boundaries. All computations were performed with TOSCA Version 6.0 on a Solbourne series 5 model 800 workstation with 64 Mbytes of physical memory. With this setup, a typical run took about 3.5 hours.

The effect of spurious boundary conditions can be understood by recalling Green's theorem. According the latter, the field due the sources outside of the region of interest is equivalent to the field surface distributions of poles and dipoles whose magnitudes are respectively proportional to the tangential and normal components of the field. One therefore expects a solution with open boundaries and a solution where spurious boundary conditions have been imposed to differ significantly only in the vicinity of the boundary. In case additional accuracy is needed near the boundary TOSCA can reconstruct the field by integrating the contribution of the coils and the induced magnetization. The effect of the boundaries is then practically eliminated. The computational cost of this procedure is very high; still, this represents a major improvement on GFUN since no iterations have to be performed to reach self-consistency.

The field profiles obtained for the three different types of end packs are illustrated in figure 2. The corresponding multipole coefficients are presented in Table 1. Note that the simple beveled end field quality is slightly worse that that of the sharp end. It is important to keep in mind that the purpose of the bevel is to reduce the dependence of the effective length on the excitation current. Figure 2 clearly illustrates the effect of the correction shims: the symmetric bumps on each side of the axis are the signature of the shims. The sextupole coefficient has become slightly positive and the field error for $x \leq 2.54 \mathrm{~cm}$ has been reduced by about an order of magnitude.

Figure 3 is a plot of $B_{y}$ as a function of the longitudinal position $z$ for different vertical offsets at $9417 \mathrm{~A}$. The origin of $z$ corresponds to the edge of the bevel. This figure reveals two interesting facts. (1) Although the field increases more rapidly as one approaches the edge of the iron, this effect is partially compensated by a faster decay. (2) The field in the center of the magnet (at $9417 \mathrm{~A}$ ) is approximately $7 \%$ weaker than what is predicted by $2 \mathrm{D}$ calculations [2]. This is apparent discrepancy is caused by the fact that the models used for the $3 \mathrm{D}$ calculations were not sufficiently long. While this may affect slightly the accuracy of the effective length prediction (especially in the case of the sharp end), the effect on the normalized sextupole and decapole is small.

As mentioned in the previous paragraph, the Main Injector dipoles magnets have parallel ends. Physically, a rectangular magnet differs from a sector magnet by two small wedge-shaped regions at both ends. These wedges act as thin quadrupoles and compensate exactly for the natural weak horizontal focusing of the sector magnet. As a result, the focusing is shifted to the vertical plane. If so desired, it is possible to correct the rectangular magnet to 
make it behave like a sector magnet. This is achieved by breaking the left-right symmetry of the correction shims. One can make all the shims identical and move the vertical symmetry axis of the shims away from the axis of the magnet, use smaller shims on one side or use a combination of both methods.

\begin{tabular}{|l||r|r|r|}
\hline I=9417 A $(150 \mathrm{GeV})$ & sharp & beveled & beveled/shims \\
\hline \hline Iron Length $(\mathrm{cm})$ & 29.845 & 29.845 & 29.845 \\
\hline Effective length $(\mathrm{cm})$ & 27.931 & 28.860 & 29.252 \\
\hline sextupole $(2.54 \mathrm{~cm})$ & -38.6 & -53.4 & +15.2 \\
\hline decapole $@ 2.54 \mathrm{~cm})$ & -10.7 & -10.5 & -13.3 \\
\hline
\end{tabular}

Table 1: The sextupole and the decapole coefficients are normalized with respect to the dipole and multiplied by $10^{4}$. For mechanical reasons, the sharp end magnet has slightly longer coils.

\section{Conclusion}

In conclusion, it is now possible to model the magnetic properties of magnet ends numerically. While this remains a time-consuming exercise, the rapid progress of the technology makes practical a system where the parameters of individual end packs are adjusted during production to minimize the effect of assembly errors. In fact, this is how the PEP interaction region quadrupoles at LBL were built in the late 70's. At the time, however, the procedure could be justified only under rather special circumstances because of its high cost.

\section{Acknowledgements}

The author would like to thank the originator of the Main Injector dipole end pack design, Mr. Stanley Snowdon, for his valuable comments.

\section{References}

[1] J. Simkin and C. W. Trowbridge, "Three-dimensional Nonlinear Electromagnetic Field Computations, using Scalar Potentials", IEE Proc.,127,6, 368-374 (1980).

[2] D.J. Harding et al., "Design Considerations and Prototype Performance of the Fermilab Main Injector Dipole", These Proceedings.

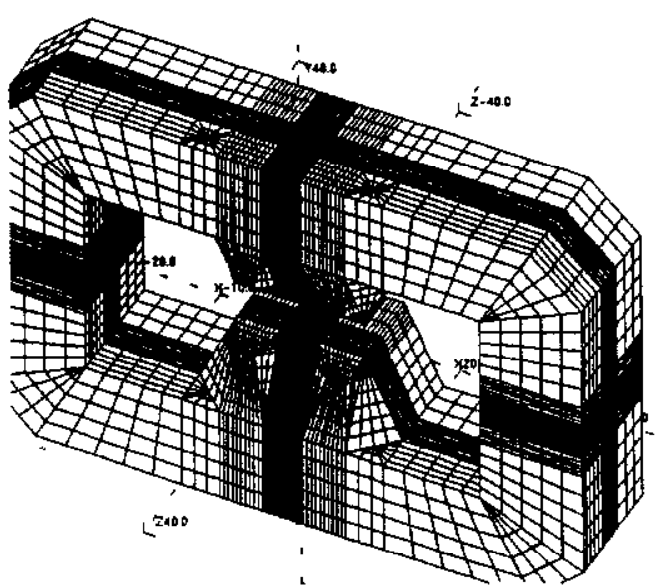

Figure 1: A beveled end with correction shims. The coils are not shown.

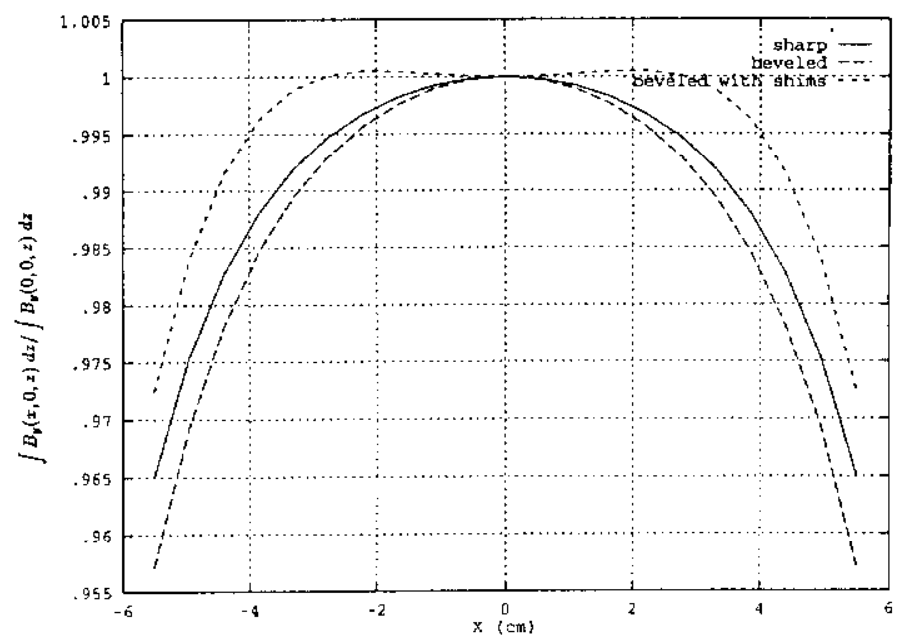

Figure 2: Integrated field profile across the aperture.

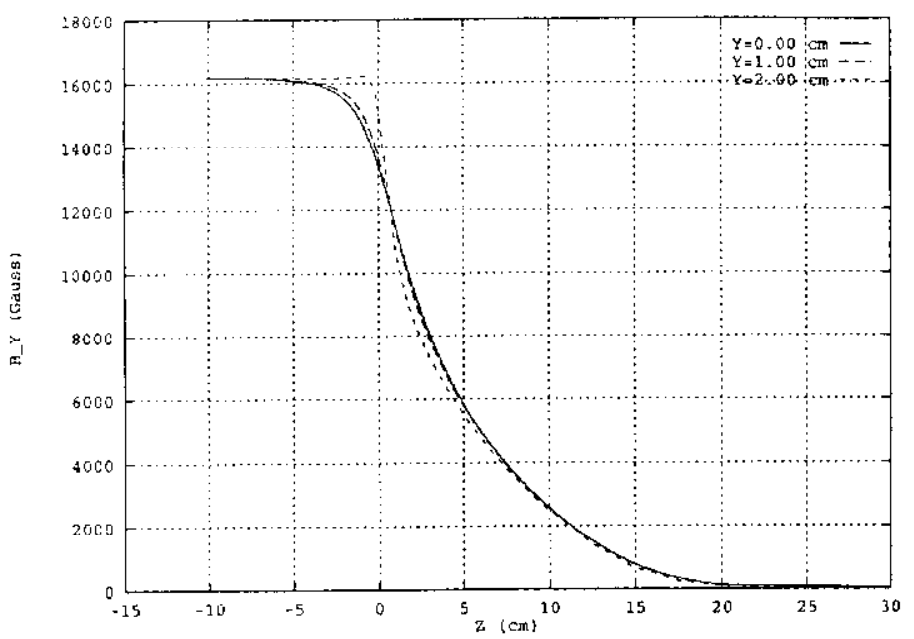

Figure 3: Vertical field vs longitudinal position at different vertical offsets for a beveled end with shims. 